\title{
Les Sapotaceae de Madagascar, deux nouvelles espèces du genre Mimusops L.
}

\author{
Richard Randrianaivo
}

\begin{abstract}
Members of the family Sapotaceae, occurring in Madagascar's various forest types, are mostly known as nanto in local dialects; some of their cultural and social values are described here. While the circumscription of Mimusops is well-defined, the delimitation of species within the genus remains unclear and their identification is often difficult. A study of herbarium specimens assigned to this genus deposited in key herbaria, two in Madagascar (TAN and TEF) and three outside the country (G, MO and P), revealed two new species: Mimusops boeniensis Randrianaivo sp. nov. and Mimusops masoalensis Randrianaivo $\mathrm{sp}$. nov. This brings the number of Mimusops species recognized in Madagascar to 16. Both of these newly recognized species are illustrated, and detailed information is provided concerning their morphology and the differences between them and other members in the genus, as well as on their ecology and distribution.
\end{abstract}

\section{RÉSUMÉ}

Les Sapotaceae de Madagascar sont rencontrés dans tous les types de forêt de l'île et sont connus sous un même nom vernaculaire sur l'ensemble du territoire, nanto. Les nanto sont importants dans la Société malgache et leur valeur culturelle est décrite ici. La circonscription taxinomique de Mimusops est bien définie. En revanche, la délimitation entre les différentes espèces au sein du genre reste confuse et l'identification des récoltes est souvent difficile. L'étude des spécimens d'herbier connus dans les herbiers nationaux (TAN et TEF) et internationaux ( $G, M O$ et $P$ ) nous a néanmoins permis de reconnaitre et de décrire deux nouvelles espèces: Mimusops boeniensis Randrianaivo sp. nov. et Mimusops masoalensis Randrianaivo sp. nov., le nombre d'espèces malgaches de Mimusops passant ainsi de 14 à 16. Cet article s'attache ensuite à fournir une description morphologique détaillée et illustrée de ces deux espèces ainsi que des renseignements sur leur distribution et leur écologie. Les différences qu'elles présentent avec les espèces les plus proches sur le plan morphologique sont par la suite discutées.

\section{INTRODUCTION}

NANTO DANS LA CULTURE, L'HISTOIRE ET L'ÉCONOMIE.

À I'exception de Chrysophyllum boivinianum (Pierre) Baehni qui est appelé Famelona, toutes les espèces malgaches de Sapotaceae sont communément appelées nanto ou nato. Le

\author{
Missouri Botanical Garden \\ Madagascar Research and Conservation Program \\ BP 3391, Antananarivo 101, Madagascar \\ E-mail: randrianaivo.richard@mobot-mg.org
}

nanto a un rôle économique, culturel et biologique important à Madagascar. Son bois est souvent cité parmi les essences utilisées dans la construction traditionnelle. Le bon bois de nanto est recherché pour l'ébénisterie, la charpente, la parqueterie, la confection des traverses de chemin de fer, de pirogues monoxyles, des pilotis, des longrines, des chapeaux de pont ainsi que de toutes parties de bâtiment (Gueneau 1971a, b). Caractérisé par une forte résistance aux activités dégradantes des microorganismes du sol, le bois de nanto est traditionnellement utilisé pour la construction de piliers de maisons. Par exemple, les piliers du Palais de la Reine de Manjakamiadana à Antananarivo ont été spécialement construits avec du bois de nanto en provenance de la forêt d'Ambinanindrano, à I'Est d'Ambositra.

Dans le domaine culturel ancestral malgache, les nanto jouent un rôle culturel particulier. Selon Boiteau (1985), ce mot vernaculaire viendrait du mot 'to' qui signifie vérité et qui ferait allusion à une propriété magique de l'arbre. Cette croyance s'illustre par exemple lors d'une cérémonie de sacrifice ou joro lors de laquelle le sang, qui occupe toujours une place importante, est parfois remplacé par l'écorce des nanto qui est généralement de couleur rouge sang. Certaines espèces de nanto font partie des plantes tinctoriales dont l'écorce a été utilisée pour la teinture dans certaines régions de Madagascar (Dubard 1914). D'où un dicton malgache « Lambamena asoka hodi-nanto, izay mamerina indroa manana ny antitra » qui signifie littéralement 'le linceul teinté deux fois de suite avec l'écorce de nanto, a une couleur rouge plus foncée'. En outre, les Sapotaceae jouent également un rôle biologique important dans les écosystèmes forestiers. La famille est surtout entomogame, la dissémination des baies sucrées est assurée par les oiseaux et certains mammifères comme les lémuriens (Judd et al. 2002). Les fruits de certaines espèces du genre Mimusops sont également vendus au marché surtout sur la côte Est de l'île.

LES SAPOTACEAE À MADAGASCAR. La famille des

Sapotaceae est représentée à Madagascar par dix genres dont quatre endémiques, et 82 espèces actuellement reconnues dont 79 sont endémiques (Madagascar Catalogue 2013). Cette famille de plantes nécessite une révision en ce qui concerne les taxons de Madagascar (Morat et Lowry 1993). Linné (1758) a décrit le genre Mimusops et les études systématiques du genre ont été multiples depuis plus de 250 ans. Les travaux menés par Aubréville (1974) sur les collections de Madagascar déposées dans I'herbier de Paris (P) pour la publication de la famille 


\section{vOLUME 8 ISSUE 2

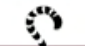 NOVEMBER 2013 MADAGASCAR 5,8 CONSERVATION \& DEVELOPMENT}

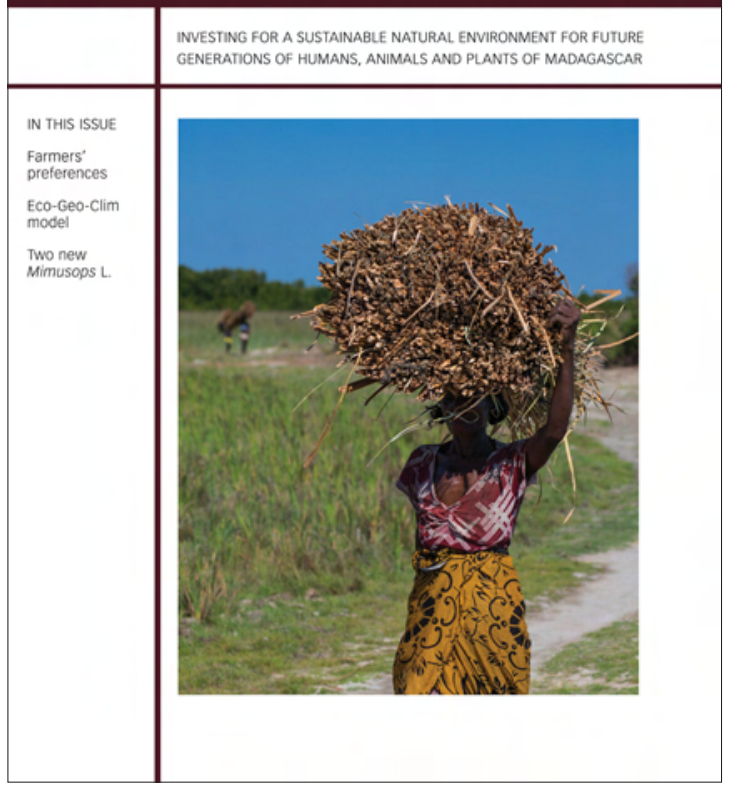

Madagascar Conservation \& Development is the journal of Indian Ocean e-Ink. It is produced under the responsibility of this institution. The views expressed in contributions to MCD are solely those of the authors and not those of the journal editors or the publisher.

All the Issues and articles are freely available at http://www.journalmcd.com

Contact Journal MCD

info@journalmcd.net for general inquiries regarding MCD funding@journalmcd.net to support the journal

Madagascar Conservation \& Development Institute and Museum of Anthropology University of Zurich

Winterthurerstrasse 190

$\mathrm{CH}-8057$ Zurich, Switzerland

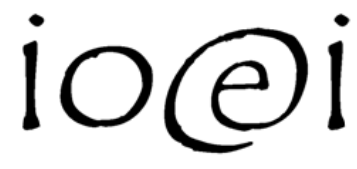

Indian Ocean e-Ink

Promoting African Publishing and Education www.ioeink.com
Missouri Botanical Garden (MBG)

Madagascar Research and Conservation Program BP 3391

Antananarivo, 101, Madagascar 
des Sapotaceae dans la Flore de Madagascar et des Comores ont révélé une grande diversité de Mimusops à Madagascar. Friedmann (1980) est le dernier à avoir décrit une nouvelle espèce malgache dans le genre, $M$. antsiranensis F. Friedmann, à partir d'un spécimen récolté en 1962 par René Capuron au sommet d'Anosiarivo, dans le massif calcaire de la Montagne des Français à Antsiranana, au Nord de Madagascar (Figure 1). Au total, 14 espèces de Mimusops sont actuellement connues de Madagascar. Le genre Mimusops appartient à la tribu des Sapoteae (Gautier 2013). Ce sont des plantes ligneuses variant de la taille de buissons à de très grands arbres. Les feuilles sont simples, alternes, insérées en spirales aux extrémités des rameaux, sans stipules. La fleur est 4-mère, le calice est formé de 8 sépales valvaires, disposés sur 2 verticilles de 4 ; la corolle est soudée à la base en un tube très court avec 8 lobes, chaque lobe est muni d'une paire d'appendices parfois laciniés à la base ; I'androcée est formé de 8 étamines épipétales, insérées au sommet du tube de la corolle, les filets sont bien distincts ; les staminodes sont également au nombre de 8, alternant avec les étamines et de même longueur, toujours pubescents sur la face externe et le rebord; I'ovaire est 8-loculaire, pubescent ou glabre, le style est grêle et long. Le fruit est une baie charnue, indéhiscente qui conserve à sa base les deux cycles de sépales, il renferme une ou plusieurs graine(s) pourvue(s) d'une petite cicatrice hilaire circulaire, basiventrale.

La présente étude décrit deux nouvelles espèces en utilisant les caractères morphologiques des différents organes, tout en mettant en relief les caractères distinctifs des espèces reconnues dans les travaux précédents, et propose une carte de distribution de ces deux espèces à partir des données de spécimens actuellement disponibles.

\section{MÉTHODES}

Les spécimens d'herbiers constituent le principal matériel d'étude (Aubréville 1972). Accompagnés de notes de terrain adéquates, ils constituent une source d'informations fondamentales (Jeffrey 1982). Malgré I'existence de méthodes modernes comme la biologie moléculaire, les études basées sur la morphologie ont été jugées suffisantes pour décrire de nouvelles espèces de Sapotaceae. Les échantillons des herbiers de MO, P, TAN et TEF ont été consultés et tous les détails végétatifs et flo-

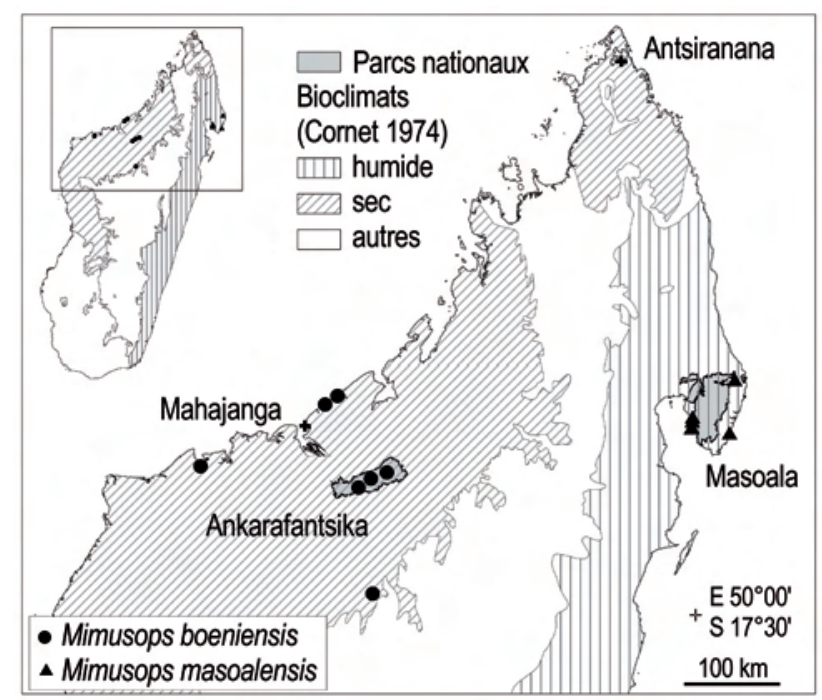

FIGURE 1. Station de récoltes des deux nouvelles espèces de Mimusops. raux ont été minutieusement examinés. Les taxons décrits ont été comparés aux espèces connues du genre. D'autres informations ont été relevées dans la base de données Tropicos (http:// www.tropicos.org) et Madagascar Catalogue (2013) du Missouri Botanical Garden pour compléter les informations issues des récoltes. Les caractères ou combinaisons de caractères spécifiques uniques ont été retenus pour distinguer les espèces les unes des autres, plus particulièrement le port de la plante, le diamètre à hauteur de poitrine (dhp en $\mathrm{cm}$ ), le type d'écorce, la forme et les dimensions du limbe foliaire, le type de nervation, le type de pétiole, la pilosité de l'ovaire, le type de pédicelle, la forme et les dimensions des fruits, le nombre et la forme de graines dans les fruits, la forme de la cicatrice hilaire et la distribution géographique.

\section{RÉSULTATS ET DISCUSSION}

MIMUSOPS BOENIENSIS RANDRIANAIVO SP. NOV.

Type : Madagascar. Région Boeny, District Ambatoboeny, Commune Andranofasika, Parc National Ankarafantsika, E046 $33^{\prime} 36^{\prime \prime}, \mathrm{S} 16^{\circ} 07^{\prime} 48^{\prime \prime}$, février 1997, fleur ; Rabevohitra 3107 (SF 34831) (holo- : TEF! TEF000877).

Paratypes: Rabevohitra 2470 (SF 33699) (TEF), Ampijoroa Station Forestière, janvier 1992, E046 $29^{\prime} 24^{\prime \prime}$, S16 10'48", fleur ; Rabevohitra 2693 (TEF), Ampijoroa Station Forestière, février 1994, E046 $29^{\prime} 24^{\prime \prime}$, S16 10'48", fruit ; Rabevohitra 2709 (TEF) ; Ampijoroa Station Forestière, février 1994, E046 $29^{\prime} 24^{\prime \prime}$, S16 $10^{\prime} 48^{\prime \prime}$, bouton floral, fleur ; Rabevohitra 2791 (TEF), Ampijoroa Station Forestière, février 1994, E046 $29^{\prime} 24^{\prime \prime}, S 16^{\circ} 10^{\prime} 48^{\prime \prime}$, bouton ; Réserves Naturelles (Ramamonjisoa) 2914 (K, MO, P, TEF), Parc National Ankarafantsika, janvier 1951, E04704'30", S1609'50", fleur ; Rakotozafy 1848A (TAN), Soalala, juillet 1997, E045 19'30", S16 06'00", fruit ; Rakotozafy 1848E (TAN), Soalala, juillet 1997, E045¹9'30", S1606'00", fruit ; Randrianaivo 646 (CNARP, MO, P, TAN), Antsahanitia-Mahajanga, mai 2001, E046 $26^{\prime} 01^{\prime \prime}, \mathrm{S} 15^{\circ} 35^{\prime} 18^{\prime \prime}$, $20 \mathrm{~m}$, fruit ; Randrianaivo 972 (CNARP, MO, P, TAN), Katsepy-Mahajanga, décembre 2003, E046 $10^{\prime} 45^{\prime \prime}$, $\mathrm{S} 15^{\circ} 49^{\prime} 14^{\prime \prime}, 46 \mathrm{~m}$, bouton, fruit ; Randrianasolo 521 (G, $\mathrm{MO}, \mathrm{P}, \mathrm{TAN})$, Ampijoroa Station Forestière, novembre 1996, E046 $49^{\prime}, \mathrm{S} 16^{\circ} 18^{\prime}, 200 \mathrm{~m}$, fruit ; Service Forestier (SF) 122 (P), janvier 1949, E046 ${ }^{\circ} 7^{\prime} 00^{\prime \prime}$, S17019'00", 480 m, fruit ; SF 10581 (Raboanary Rajaona 43) (P), Marosakoa, août 1954, E046²9'24", S16 10'48", fruit ; SF (Capuron) 11992 (G, P, TEF), Ankarafantsika, mai 1957, E046 $33^{\prime} 36^{\prime \prime}-E 047^{\circ} 08^{\prime} 24^{\prime \prime}$, S15 $35^{\prime} 24^{\prime \prime}-S 16^{\circ} 12^{\prime} 00^{\prime \prime}$, fruit ; SF 24315 (Capuron) (TEF), Ampasimaniry-Mahajanga, novembre 1965, E046 ${ }^{\circ} 30^{\prime} 00^{\prime \prime}, \mathrm{S} 15^{\circ} 31^{\prime} 00^{\prime \prime}, 0$ m, fruit.

Diagnose: Tree with small obovate leaves less than $6.5 \mathrm{~cm}$ long, glabrous ovary, small ellipsoid fruit $(18-20 \mathrm{~mm}$ $x$ 36-40 mm) monospermous, and persistent reflexed calyx (Figure 2).

Description : Arbre de 2,5 m à $12 \mathrm{~m}$ de hauteur ; diamètre à hauteur de poitrine (dhp) de 5 à $15 \mathrm{~cm}$; écorce gris-noir crevassée longitudinalement. Présence de latex blanc dans toutes les parties. Feuilles elliptiques à obovées, glabres, obtuses à arrondies au sommet, cunéiformes à la base; limbe de 3,5-6 × 1,5-3 cm ; nervation réticu- 
lée, généralement finement marquée sur les deux faces ou parfois légèrement effacée sur la face supérieure; pétiole grêle, 5-15 mm de long x 1-1,2 mm de section, pubescent. Fleurs fasciculées à l'aisselle des feuilles ; pédicelle de $1 \mathrm{~cm}$ de long environ; lobes des sépales extérieurs long de 5-6 mm, pubescent extérieurement; pétales, étamines, et staminodes tôt caduques. ; ovaire glabre. Fruit ellipsoïde, 18-20 mm x 36-40 mm, apiculé, contenant une seule graine $(22-24 \times 15-16 \times 13-14$ $\mathrm{mm})$ pourvue d'une cicatrice basiventrale $(6 \times 5 \mathrm{~mm})$; pédicelle $12-15 \times 1,2 \mathrm{~mm}$, calice révoluté sur les fruit.

Floraison : janvier-février.

Fructification : mars-novembre.

Noms vernaculaires : nato, nanto

Étymologie : le nom fait référence à la toponymie de la localité du spécimen type, la région Boeny.

Particularités: Mimusops boeniensis (Figure 2) est caractérisé par la glabrescence de l'ovaire qui la différencie de la plupart des espèces du genre. $M$. boeniensis et M. occidentalis sont deux espèces connues pour le caractère glabrescent de leurs ovaires et rencontrées dans le bioclimat sec (sensu Cornet 1974). Néanmoins M. boeniensis se distingue par la taille et la forme de ses feuilles et de ses fruits. Chez M. boeniensis, les feuilles sont relativement petites et obovées avec un limbe de moins de $6,5 \mathrm{~cm}$ de longueur, courtement pétiolées, les fruits sont petits, pyriformes inversés et monospermes, alors que chez M. occidentalis, les feuilles sont assez grandes, étroitement oblongues avec un limbe de plus de $7,5 \mathrm{~cm}$ de longueur et un pétiole long et grêle ; les fruits sont plus grands, sphériques à ovoïdes et souvent polyspermes. II est possible qu'il existe des hybrides avec M. capuronii var. retusa Aubrév. dans I'Ankarana (L. Gautier In litt.).

Statut de conservation: Mimusops boeniensis a une aire de distribution restreinte, à cheval sur les régions Boeny et Betsiboka. Quatre des cinq sous-populations connues sont distribuées en dehors du réseau des Aires Protégées et une seule a été recensée dans le Parc National Ankarafantsika (Figure 1). Le passage presque régulier de feux de brousse dans ce parc n'assure pas une protection suffisante à cette sous-population. Avec une aire d'occurrence (EOO) de I'ordre de $19300 \mathrm{~km}^{2}$, une aire d'occupation (AOO) de $63 \mathrm{~km}^{2}$ et n'étant présente que dans quatre stations (locations), M. boeniensis est évalué comme En Danger EN B2ab (ii, iii, iv, v). par application des catégories et critères de la Liste Rouge de I'IUCN (2012).

\section{MIMUSOPS MASOALENSIS RANDRIANAIVO SP. NOV.}

Type : Madagascar, Région Analanjorofo, District Maroantsetra, forêt littorale de Tampolo, Parc National Masoala, 18 octobre 2001, E04957'38", S1543'45", $10 \mathrm{~m}$ d'altitude, fruit, Randrianaivo 703, (holo- : TAN! TAN001987; iso- : MO).

Paratypes : Aridy 512 ; Labat 3421 (MO, P, TEF), AndranobeMasoala, octobre 2001, E04957'54", S1540'20", 270 m; Rabevohitra 3309 (SF 35066) (TEF), Tanambao-Masoala,

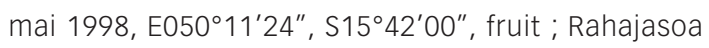
1120 (TAN), Andranobe-Masoala, mars 1995, E0495',

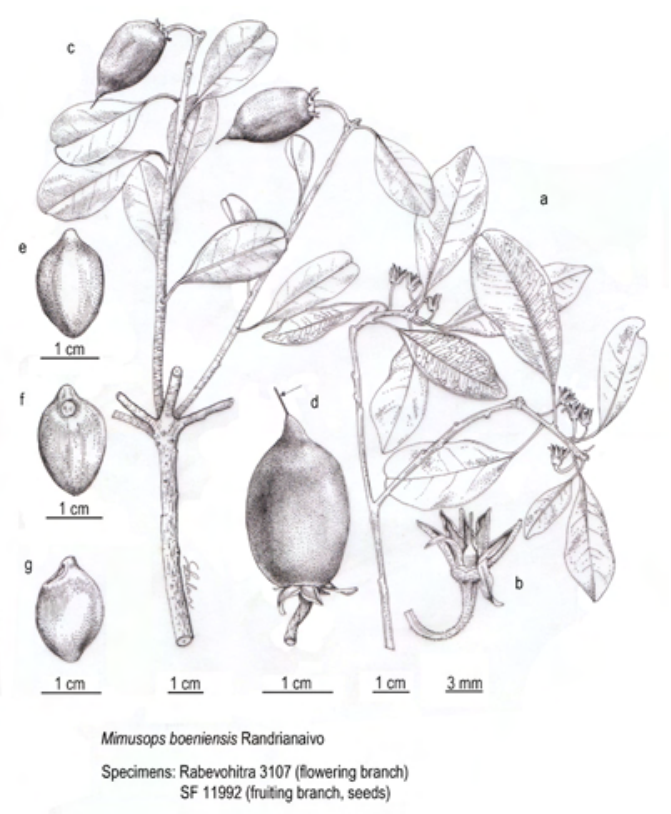

FIGURE 2. Mimusops boeniensis Randrianaivo. (a : rameau florifère, $b$ : fleur, $c$ : rameau fructifère, $d$ : fruit, e : graine vue dorsale, $f$ : graine vue ventrale, $\mathrm{g}$ : graine vue latérale, dessins Roger Lala Andriamiarisoa)

S15³8'S, fruit ; Randrianaivo 104 (G, MO, P, TAN), Saha-

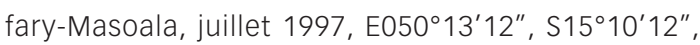
fruit ; Randrianaivo 109 (G, MO, P, TAN), Sahafary-Masoala, juillet 1997, E050¹3'12", S15¹0'12", fruit ; Randrianaivo 115 (G, MO, P, TAN), Sahafary-Masoala, juillet 1997, E050¹3'12", S15¹0'12", fruit ; Randrianaivo 667 (G, MO, P, TAN), Tampolo-Masoala, octobre 2001, E04957'24", S1544'12", 200-400 m, fruit ; Schatz 3022 (G, MO, P, TAN), Ambanizana, Masoala, décembre 1990, E049³4'48", S15 22'48", 0-10 m, fruit ; Vasey, N. 240 (G, MO, P, TAN), Andranobe-Masoala, décembre 1994, E049 $57^{\prime} 51^{\prime \prime}, \mathrm{S} 15^{\circ} 40^{\prime} 24^{\prime \prime}, 110-260 \mathrm{~m}$, fruit ; Vasey, N. 321 (G, MO, P, TAN), Andranobe-Masoala, janvier 1995, E049 $57^{\prime} 51^{\prime \prime}, \mathrm{S} 15^{\circ} 40^{\prime} 24^{\prime \prime}, 110-260 \mathrm{~m}$, fruit. Diagnose : Tree with large leathery leaves, limb simple, long petioles $(5-6 \mathrm{~cm})$, rare fruit on flowering branches, sepals having an outer hull (Figure 3 ).

Description : Arbre de 12-35 m de hauteur ; diamètre à hauteur de poitrine (dhp) 50-65 cm ; présence de latex blanc dans toutes les parties, écoulement de latex relativement faible par rapport aux autres espèces du genre ; écorce gris-noir crevassée longitudinalement et rouge à l'intérieur; parties jeunes recouvertes de poils roux. Feuilles $14-36 \times 7-16 \mathrm{~cm}$; limbes obovés ou oblongues, arrondis, légèrement émarginés au sommet, cunéiformes à la base, épais et coriaces, initialement velus brun-argenté sur les faces inférieures, puis glabrescents ; nervation secondaire réticulée, généralement distincte sur la face inférieure, parfois légèrement effacée sur la face supérieure ; pétioles robustes, longs jusqu'à 5-6 cm, environ trois fois plus longs que les pédicelles fructifères. Fleur inconnue. Fruit ovoïde ou ellipsoïde (4,5-7 cm x 2,5-3,5 $\mathrm{cm})$, brun clair, arrondi et parfois apiculé au sommet, inséré à l'aisselle des feuilles ou de leurs cicatrices, renfermant une graine à testa vernissé et pourvu d'une petite cicatrice hilaire circulaire basiventrale, rugueuse 
et mate contrastant avec le brillant du reste de la graine ; calice persistant sur les fruits, brun, sépales extérieurs nettement carénés mesurant 10 mm; pédicelle robuste, $2 \mathrm{~cm}$ de long environ.

Floraison : inconnue.

Fructification : septembre-juillet.

Noms vernaculaires : nanto voarantoala, voaranto

Étymologie : le nom fait référence à la toponymie de la localité du type, la presqu'île Masoala (Figure 1).

Distribution : Mimusops masoalensis (Figure 3) est rencontré sur la presqu'île Masoala, dans le Nord-Est de Madagascar (Figure 1), dans une forêt dense humide de basse altitude depuis le niveau de la mer jusqu'à $400 \mathrm{~m}$ d'altitude.

Particularités: Mimusops masoalensis (Figure 3) se distingue des autres espèces de Mimusops de Madagascar par la combinaison de caractères suivants : grandes feuilles coriaces, à pétiole long (5 à $6 \mathrm{~cm}$ ) et à marge simple, et un nombre réduit de fruits sur les rameaux florifères, les fruits aux sépales présentant une carène extérieure. Sur le terrain, M. masoalensis peut être confondu avec M. voalela, M. longepedicellata ou M. coriacea par la taille des feuilles, mais s'en distingue par son pédicelle plus court et son pétiole relativement court. M. antongilensis est aussi une espèce de la presqu'île Masoala proche de M. masoalensis par ses feuilles relativement grandes et coriaces. M. masoalensis s'en distingue par ses feuilles plus larges et le bord du limbe simple.

Statut de conservation: Mimusops masoalensis n'est connu que de la presqu'ile Masoala. Son aire d'occurrence (EOO) est de $292 \mathrm{~km}^{2}$ et son aire d'occupation (AOO) de $45 \mathrm{~km}^{2}$. Quatre sous-populations sont connues dont deux seulement sont distribuées dans l'aire protégée de Masoala. Dans la mesure où cette région est sous la menace des cyclones, $M$. masoalensis est classé En Danger EN B1ab (i, ii, iii, iv) + 2ab (i, ii, iii, iv) selon les catégories et les critères de la Liste Rouge de l'IUCN (2012).

\section{REMERCIEMENTS}

Mes plus vifs remerciements vont à toutes les personnes et institutions qui, de près ou de loin, ont contribué à la réalisation de cette publication, en particulier Pete Lowry, Christian Camara, Chris Birkinshaw, Sylvie Andriambololonera et Marina Rabarimanarivo. Je tiens également à remercier les deux rapporteurs anonymes et Laurent Gautier qui m'ont permis d'améliorer sensiblement cette contribution. Mes remerciements vont aussi aux responsables des herbiers TAN, TEF, $\mathrm{P}$ et MO, à Fano Rajaonary qui a réalisé la Figure 1, et Roger Lala Andriamiarisoa, pour les illustrations des deux nouvelles espèces décrites ici.

\section{RÉFÉRENCES}

Aubréville, A. 1972. Gambeyobotrys, genre nouveau de Sapotacées. Adansonia, sér. 2, 12, $2: 187-189$.

Aubréville, A. 1974. Sapotaceae. Flore de Madagascar et des Comores, famille 164. Muséum national d'Histoire naturelle, Paris. 128 pp.

Boiteau, P. 1985. Dictionnaire des noms malgaches de végétaux. Microédition N. 850410. Archives et Documents. Muséum national d'Histoire naturelle, Paris.

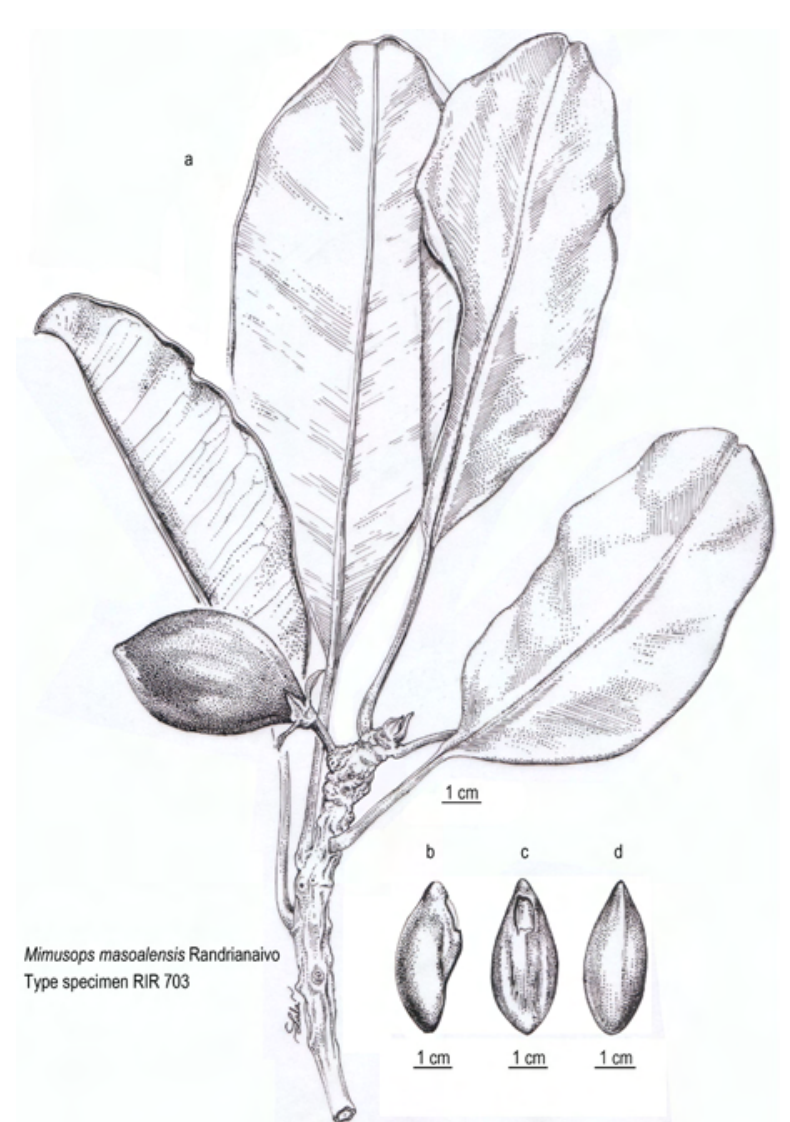

FIGURE 3. Mimusops masoalensis Randrianaivo. (a : rameau fructifère, $b$ : graine vue latérale, $c:$ graine vue ventrale, $d:$ graine vue dorsale, dessins Roger Lala Andriamiarisoa)

Cornet, A. 1974. Essai cartographique bioclimatique à Madagascar. Notice explicative N. 55. ORSTOM, Paris.

Dubard, M. 1914. Description de quelques Manilkara. Notulae Systematicae 3: $45-47$.

Friedmann, F. 1980. Une espèce nouvelle du genre Mimusops (Sapotaceae) à Madagascar. Adansonia, sér. 2, 20, 2: 229-233.

Gautier, L., Naciri, Y., Anderberg, A. A., Smedmark, J. E. E., Randrianaivo, R. \& Swenson, U. 2013. A new species, genus and tribe of Sapotaceae, endemic to Madagascar. Taxon 62: 972-983. (doi:10.12705/625.17)

Gueneau, P. 1971a. Bois de Madagascar, Possibilités d'Emplois. Centre Technique Forestier Tropical, Tananarive, 75pp.

Gueneau, P. 1971b. Bois et Essences Malgaches (Supplément). Centre Technique Forestier Tropical, Tananarive, 65pp.

IUCN 2012. IUCN Red List Categories and Criteria: version 3.1. IUCN Species Survival Commission. IUCN, Gland and Cambridge.

Jeffrey, C. 1982. An Introduction to Plant Taxonomy. Cambridge University Press, Royal Botanical Gardens, Kew.

Judd, W. S., Campbell, C. S., Kellogg, E. A. et Stevens P. 1999. Botanique Systématique, Une Perspective Phylogénétique. Université De Boeck.

Madagascar Catalogue. 2013. Catalogue of the Vascular Plants of Madagascar. Missouri Botanical Garden, St. Louis, U.S.A. \& Antananarivo, Madagascar <http://www.efloras.org/madagascar> consulté le 1er septembre 2013.

Morat, P. \& Lowry II, P. P. 1993. Do we really know as much as we think about the flora of the Africa-Madagascar region? V International Botanical Congress, Yokohama, Abstract 33.

\section{SUPPLEMENTARY MATERIAL.}

AVAILABLE ONLINE ONLY.

FIGURE S1. Holotype of Mimusops boeniensis Randrianaivo.

FIGURE S2. Holotype of Mimusops masoalensis Randrianaivo.

TABLE S1. Acronyms of the main herbaria holding specimens collected in Madagascar. 